\title{
ISS Properties of Nonholonomic Vehicles
}

\author{
Herbert G. Tanner \\ Mechanical Engineering Dept., University of New Mexico, Albuquerque, NM 87131
}

\begin{abstract}
The paper presents the first result on nonholonomic systems enjoying Input to State Stability (ISS) properties. Although it is known that smooth stabilizability implies ISS, the converse is not generally true. This leaves the possibility of non smoothly stabilizable systems being ISS with respect to a particular input, after an appropriate feedback transformation. This is shown to be true for the case of the unicycle with a dynamic extension, in a particular topology induced by a metric appropriate for this type of systems. A feedback control law renders the closed loop system locally ISS in the particular topology. Potential applications include stability and robustness analysis of formations of mobile robots.
\end{abstract}

\section{Introduction}

Input-to-state stability (ISS) [1] is a framework for stability and robustness analysis that has proved extremely useful in a variety of applications, from PWM control systems [2] to formation control and robotics [3]. The success of input-to-state stability as an analysis tool is in part due to its invariance properties under a large class of system interconnections [1].

Lately there has been related work on applications of input-to-state stability on vehicle formations [3]. Taking advantage of the invariance properties of ISS, one can compute error bounds for the vehicles in the formation, which depend on the formation leader input [4]. When the system dynamics is linear, the approach provides recursive matrix equations that yield the gains from input to error, for the whole formation as well as for interconnected subgroups. However, vehicles are usually modeled as nonholonomic systems. It is thus

Email address: tanner@unm.edu (Herbert G. Tanner).

URL: http://www .unm. edu/ tanner (Herbert G. Tanner). 
natural to ask if such techniques can be applicable to the case of nonholonomic dynamics.

The highly nonlinear nature of the problem and the fact that nonholonomic systems cannot be stabilized by continuous static state feedback [5], make the problem particularly challenging. Numerous approaches to nonholonomic stabilization to equilibrium points are based either on discontinuous or timevarying techniques $[6,7]$. Although ISS is related to smooth stabilizability, in the sense that the latter implies the former [8], there has been no result suggesting equivalence. Motivated by the possibility of establishing local ISS properties for systems that are not smoothly stabilizable, this paper investigates the case where a vehicle described by unicycle kinematics with a dynamic extension, is required to follow a moving target. It seems that the dynamic extension in the vehicle dynamics plays a significant role in the ability to establish its local ISS properties with respect to acceleration disturbances injected by the moving target. The approach presented capitalizes on the fact that these disturbances enter in a linear fashion into the vehicle dynamics. The stability analysis exploits a topology induced by a particular nonholonomic metric after an input feedback transformation. With respect to this metric, the vehicle dynamics are shown to be exponentially stable, opening the way for the establishment of local ISS properties. Similar nonholonomic metrics have been used for characterizing shortest paths $[9,10]$ for mobile robots. A singular perturbation analysis then provides the necessary controller gains that ensure asymptotic stability (exponential in the new metric) for the nominal system and input-to-state stability for the perturbed. In the metric-induced topology, the gain function from disturbance to error is linear, allowing a computationally efficient propagation of the ISS gains through vehicle interconnections as those described in [4].

The rest of the paper is organized as follows: in Section 2 we give a brief description of the problem and we introduce the nonholonomic metric. Section 3 introduces the feedback controller and establishes its stability properties using singular perturbations analysis. In Section 4 the closed loop system is shown to be locally ISS with respect to acceleration input disturbances. Section 5 verifies the paper's results via numerical simulations. Finally, Section 6 elucidates the contributions of this paper.

\section{Problem Description}

Vehicles in formation are generally required to maintain a desired distance and bearing from each other [11]. In such a scenario, a vehicle such as the one depicted in Figure 1, regulates its distance $r$ and its bearing $\varphi$ with respect to another, leading vehicle. Let us attach a reference frame at the desired position 


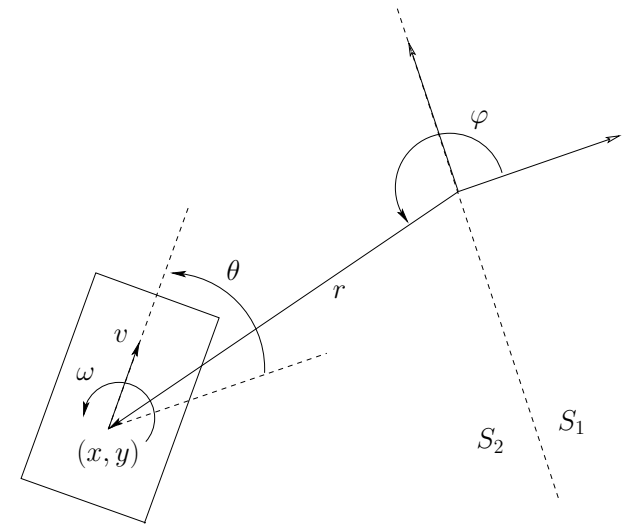

Fig. 1. A vehicle tracking a moving target.

of the following vehicle, aligned with the desired orientation. The configuration of the following vehicle with respect to that frame can be specified by the position offset, $(x, y)$, and the orientation offset, $\theta$. The following vehicle moves with translational speed $v$ and rotational velocity $\omega$. It is controlled via its translational acceleration $a$ and rotational acceleration $\alpha$ inputs, respectively. The dynamics of the following vehicle are given as:

$$
\begin{aligned}
& \dot{x}=v \cos \theta \\
& \dot{y}=v \sin \theta \\
& \dot{\theta}=\omega \\
& \dot{v}=a \\
& \dot{\omega}=\alpha
\end{aligned}
$$

This model is essentially equivalent to the one derived in [12]. Assume now that a kinematic controller on the following vehicle can specify a reference speed $v_{d}$ and rotational velocity $\omega_{d}$, in order for the offset $(x, y, \theta)$ to converge to zero asymptotically. These reference velocity inputs have to be realized through the translational and rotational acceleration inputs, $a$ and $\alpha$, respectively. Let $e_{v}$ and $e_{\omega}$ denote the velocity errors, the difference between the vehicle velocities and the reference velocity inputs. Then the dynamics can be rewritten as:

$$
\begin{aligned}
\dot{x} & =\left(v_{d}+e_{v}\right) \cos \theta \\
\dot{y} & =\left(v_{d}+e_{v}\right) \sin \theta \\
\dot{\theta} & =\omega_{d}+e_{\omega} \\
\dot{e}_{v} & =a \\
\dot{e}_{\omega} & =\alpha .
\end{aligned}
$$

Since the control specifications are prescribed in terms of desired distance and bearing, the use of polar coordinates seems more appropriate:

$$
r \triangleq \sqrt{x^{2}+y^{2}}, \quad \varphi \triangleq \arctan (y / x), \quad e_{\theta} \triangleq \theta-2 \varphi .
$$


In polar coordinates the system dynamics take the following form:

$$
\begin{aligned}
\dot{r} & =\left(v_{d}+e_{v}\right) \cos \left(e_{\theta}+\varphi\right) \\
\dot{\varphi} & =\frac{v_{d}+e_{v}}{r} \sin \left(e_{\theta}+\varphi\right) \\
\dot{e}_{\theta} & =\omega_{d}+e_{\omega}-2\left(v+e_{v}\right) \sin \left(e_{\theta}+\varphi\right) \\
\dot{e}_{v} & =a \\
\dot{e}_{\omega} & =\alpha .
\end{aligned}
$$

Let us consider the regions, $S_{1}=\{(r, \varphi, \theta, v, \omega) \mid \cos \varphi>0\}$ and $S_{2}=$ $\{(r, \varphi, \theta, v, \omega) \mid \cos \varphi<0\}$ (Figure 1), and restrict our analysis to the domain $S_{1} \cup S_{2}$. Note, that by the definition of $\varphi$, the origin belongs to $S_{1}$. When the inputs have to be bounded, the analysis has to be restricted to $S_{1}^{\delta}=\{(r, \varphi, \theta, v, \omega) \mid \cos \varphi>\delta\}$ and $S_{2}^{\delta}=\{(r, \varphi, \theta, v, \omega) \mid \cos \varphi<-\delta\}$, for a sufficient $\delta>0$. Due to the invariance of $S_{1}$ and $S_{2}$, which will be established shortly (Section 3.2), it makes sense to define the following metric on $S_{1}$ and $S_{2}$ :

Proposition 2.1 The function $d_{1}: S_{i} \rightarrow \mathbb{R}, i=1,2$ given as:

$$
d_{c}\left(r, \varphi, \theta, e_{v}, e_{\omega}\right) \triangleq \sqrt{\frac{r^{2}}{\cos ^{2} \varphi}+\sin ^{2} \varphi+\theta^{2}+e_{v}^{2}+e_{\omega}^{2}}
$$

defines a metric on $S_{i}$.

Proof. Function (4) has to satisfy: (i) $d_{c}(z) \geq 0, \forall z \in S_{i}$, (ii) $d_{c}(z)=0 \Leftrightarrow$ $z=0, \forall z \in S_{i}$, and (iii) $d_{c}\left(z_{1}+z_{2}\right) \leq d_{c}\left(z_{1}\right)+d_{c}\left(z_{2}\right), \forall z_{1}, z_{2} \in S_{i}$. We will show that this is true for $S_{1}$; the case for $S_{2}$ follows similarly. Properties (i)(ii) are straightforward. For (iii), consider any two vectors in $S_{1}, z_{1}$ and $z_{2}$. Then (iii) becomes obvious in Cartesian coordinates by taking the difference $d_{c}\left(z_{1}+z_{2}\right)-d_{c}\left(z_{1}\right)-d_{c}\left(z_{2}\right)$. In $S_{1}$, for this difference to be negative, it suffices to show that $\frac{r}{\cos \varphi}-\frac{r_{1}}{\cos \varphi_{1}}-\frac{r_{2}}{\cos \varphi_{2}}<0$. Substituting:

$$
\frac{r}{\cos \varphi}-\frac{r_{1}}{\cos \varphi_{1}}-\frac{r_{2}}{\cos \varphi_{2}}=-\frac{\left(x_{2} y_{1}-x_{1} y_{2}\right)^{2}}{x_{1} x_{2}\left(x_{1}+x_{2}\right)}
$$

which is negative in $S_{1}$ since $x_{1}, x_{2}>0$. Similarly it is shown for $S_{2}$.

Figure 2 shows the topology induced by the metric on the $(x, y)$ plane for a constant $\theta$. Note that this metric is valid on both $S_{1}$ and $S_{2}$, but not in $S_{1} \cup S_{2}$. Our stability results will be expressed with respect to this metric.

The goal in this paper is to establish Input-to-State Stability (ISS) properties for system (1). Input-to-State Stability is defined as follows [13]: 


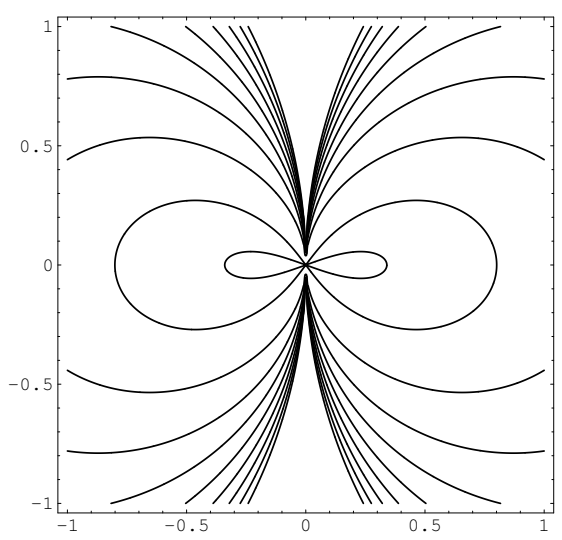

Fig. 2. The $(x, y)$-topology induced by the metric for a constant $\theta$.

Definition 2.2 (ISS) Consider a system of the form $\dot{x}=f(x, y)$, evolving in finite-dimensional spaces $\mathbb{R}^{n}$ with inputs $u \in \mathbb{R}^{m}$ that are measurable locally essentially bounded. Assume that $f: \mathbb{R}^{n} \times \mathbb{R}^{m} \rightarrow \mathbb{R}^{n}$ is locally Lipschitz and satisfies $f(0,0)=0$. The system is input to state stable if

$$
\|x(t)\| \leq \beta(\|x(0)\|, t)+\gamma\left(\sup _{t \geq 0}\|u\|\right)
$$

for some $\beta \in \mathcal{K} \mathcal{L}$ and $\gamma \in \mathcal{K}_{\infty}$, and for all $t \geq 0$.

\section{Closed Loop Stability}

\subsection{The Singularly Perturbed System}

With an appropriate choice of control inputs and sufficiently large gains, (3) can be transformed into a singularly perturbed system. To this end, let the reference velocities be defined as:

$$
\begin{aligned}
v_{d} & =-\frac{k_{1} r}{\cos \varphi} \\
\omega_{d} & =2\left(v_{d}+e_{v}\right) \sin \left(e_{\theta}+\varphi\right)-k_{\omega} e_{\theta},
\end{aligned}
$$

corresponding to the output of a kinematic controller designed to stabilize (1a)-(1c). The acceleration inputs are then designed so that the actual velocities track the reference inputs:

$$
\begin{aligned}
& a=-k_{2} e-\frac{r^{2} \sec ^{3} \varphi+\cos \varphi \sin ^{2} \varphi}{r} \\
& \alpha=-e_{\theta}-k_{\alpha} e_{\omega} .
\end{aligned}
$$


If we let $k_{\alpha}=k_{\omega} k_{\theta}$, with $k_{\theta}>1$ and substitute (5b) and (6b) into (3) we obtain a singular perturbed version of (3), in which the boundary layer system is given as

$$
\frac{1}{k_{\omega}}\left[\begin{array}{l}
\dot{e}_{\theta} \\
\dot{e}_{\omega}
\end{array}\right]=-\left[\begin{array}{cc}
1 & -\frac{1}{k_{\omega}} \\
\frac{1}{k_{\omega}} & k_{\theta}
\end{array}\right]\left[\begin{array}{l}
e_{\theta} \\
e_{\omega}
\end{array}\right] .
$$

Letting $\tau=t / \epsilon$ and $\epsilon \triangleq \frac{1}{k_{\omega}}$, it is easily seen that the boundary layer system is exponentially stable:

$$
\frac{\mathrm{d} e_{\theta}}{\mathrm{d} \tau}=-e_{\theta}, \quad \frac{\mathrm{d} e_{\omega}}{\mathrm{d} \tau}=-k_{\theta} e_{\omega}
$$

The reduced system then takes the form:

$$
\begin{aligned}
\dot{r} & =\left(v_{d}+e_{v}\right) \cos \varphi \\
\dot{\varphi} & =\frac{v_{d}+e_{v}}{r} \sin \varphi \\
\dot{e}_{v} & =a
\end{aligned}
$$

Applying (5a) and (6a) in (8), the closed loop reduced system becomes:

$$
\begin{aligned}
\dot{r} & =e_{v} \cos \varphi-k_{1} r \\
\dot{\varphi} & =\frac{e_{v} \sin \varphi}{r}-k_{1} \tan \varphi \\
\dot{e}_{v} & =-k_{2} e_{v}-\frac{r^{2} \sec ^{3} \varphi+\cos \varphi \sin ^{2} \varphi}{r}
\end{aligned}
$$

Equations (9) are exponentially stable with respect to the metric (4). Consider the Lyapunov function:

$$
V_{r}\left(r, \varphi, e_{v}\right) \triangleq \frac{r^{2}}{\cos ^{2} \varphi}+\sin ^{2} \varphi+e_{v}^{2}
$$

Its time derivative satisfies:

$$
\dot{V}_{r}=-2 k_{2} e_{v}^{2}-2 k_{1}\left(\frac{r^{2} \sec ^{2} \varphi}{\cos ^{2} \varphi}+\sin ^{2} \varphi\right) \leq-2 \min \left\{k_{1}, k_{2}\right\} d_{c}^{2}\left(r, \varphi, e_{v}\right)
$$

From the exponential stability of (7) and (8) it follows that there is a sufficiently large $k_{\omega}$, for which (3) is exponentially stable with respect to the metric (4). An estimate for the required lower bound for $k_{\omega}$ is derived in the following Section. 


\subsection{Gain Selection for Stability}

The stability analysis will be performed for the case of $\cos \varphi>0$. Case $\cos \varphi<$ 0 can be treated similarly. In the course of the discussion it will become clear that either one of these regions is made positively invariant through (6a)-(6b). Consider the following Lyapunov function for the boundary layer system (7):

$$
V_{b}\left(e_{\theta}, e_{\omega}\right) \triangleq \frac{1}{2} e_{\theta}^{2}+\frac{1}{2 k_{\theta}} e_{\omega}^{2} .
$$

Combining (11) with (10) we define a Lyapunov function for the singular perturbed system:

$$
V=r^{2} \sec ^{2} \varphi+\sin ^{2} \varphi+e_{v}^{2}+\frac{1}{2}\left(e_{\theta}^{2}+\frac{e_{\omega}^{2}}{k_{\theta}}\right)
$$

Its time derivative, $\dot{V}$ is:

$$
\begin{gathered}
\dot{V}=-\frac{k_{\theta}-1}{2 k_{\theta}}\left(e_{\theta}-e_{\omega}\right)^{2}-\frac{1+2 k_{\alpha}-k_{\theta}}{2 k_{\theta}}\left(e_{\theta}^{2}+e_{\omega}^{2}\right)+\frac{e_{v} \sin (2 \varphi)\left[\sin \left(\varphi+e_{\theta}\right)-\sin \varphi\right]}{r} \\
+2 k_{1} \cos e_{\theta} \sin ^{2} \varphi-k_{1} \sin (2 \varphi) \sin e_{\theta}-2 k_{2} e_{v}^{2} \\
-2 e_{v} r \sec ^{3} \varphi \sin ^{2}\left(\frac{e_{\theta}}{2}\right)-2 k_{1} r^{2} \cos e_{\theta} \sec ^{4} \varphi .
\end{gathered}
$$

Using the fact that $\left|e_{\theta}\right| \leq \frac{\pi}{2}$, implying $\cos e_{\theta} \geq 0$ and $\left|e_{\theta}\right| \geq\left|\sin e_{\theta}\right|$, we can bound $\dot{V}$ as follows:

$$
\begin{aligned}
& \dot{V} \leq-\frac{k_{\theta}-1}{2 k_{\theta}}\left(e_{\theta}-e_{\omega}\right)^{2}-\frac{1+2 k_{\alpha}-k_{\theta}}{2 k_{\theta}}\left(e_{\theta}^{2}+e_{\omega}^{2}\right) \\
&-2 \min \left\{k_{1}, k_{2}\right\} \cos e_{\theta}\left(e_{v}^{2}+r^{2} \sec ^{4} \varphi+\sin ^{2} \varphi\right) \\
&+2 \max \left\{1, k_{1}\right\}\left|e_{\theta}\right| \cos \varphi\left(1+\frac{\left|e_{v}\right|}{r}\right)\left(|\sin \varphi|+r^{2} \sec ^{4} \varphi\right) .
\end{aligned}
$$

From the local Lipschitz continuity of $g(x)=x^{2}$ it follows that there exists a positive constant $L$ such that $\left|r^{2} \sec ^{4} \varphi\right| \leq L\left|r \sec ^{2} \varphi\right|=L r \sec ^{2} \varphi$, from which we can extend the bound of $\dot{V}$ :

$$
\begin{aligned}
& \dot{V} \leq-\frac{k_{\theta}-1}{2 k_{\theta}}(\left.e_{\theta}-e_{\omega}\right)^{2}-\frac{1+2 k_{\alpha}-k_{\theta}}{2 k_{\theta}}\left(e_{\theta}^{2}+e_{\omega}^{2}\right) \\
&-2 \min \left\{k_{1}, k_{2}\right\} \cos e_{\theta}\left(e_{v}^{2}+r^{2} \sec ^{4} \varphi+\sin ^{2} \varphi\right) \\
&+2(1+L) \max \left\{1, k_{1}\right\}\left|e_{\theta}\right| \cos \varphi\left(1+\frac{\left|e_{v}\right|}{r}\right)\left(|\sin \varphi|+r \sec ^{2} \varphi+\left|e_{v}\right|\right) .
\end{aligned}
$$

Lemma 3.1 The term $\frac{e_{v}}{r}$ is upper bounded by a positive constant c.

Proof. Boundedness of $\frac{e_{v}}{r}$ follows from the stability of (9). If $\frac{1}{k_{2}}$ is treated as a singular parameter then (9) is transformed to a boundary layer system $\frac{d e_{v}}{d \tau}=-e_{v}$ and a reduced system:

$$
\dot{r}=-r k_{1} \quad \dot{\varphi},=-k_{1} \tan \varphi,
$$


which is also exponentially stable. Therefore, for a sufficiently large $k_{2}$, the origin of (9) is exponentially stable. The time scale decomposition of (9) induced by the increased $k_{1}$ ensures that $\left|e_{v}\right|$ reduces much faster than $r$, making the ratio $\frac{\left|e_{v}\right|}{r}$ converge to zero exponentially. This exponential convergence implies that there are positive constants, $c$ and $m$ such that

$$
\frac{\left|e_{v}\right|}{r} \leq c e^{-m t} \Rightarrow \frac{\left|e_{v}\right|}{r} \leq c, \quad \forall t \geq 0 .
$$

Using the bound on $\frac{\left|e_{v}\right|}{r}$ suggested by the aforementioned Lemma, we can obtain the following bound for $\dot{V}$ :

$$
\dot{V} \leq-\lambda_{\min }(Q) d_{c}^{2}\left(r, \varphi, e_{v}, e_{\theta}, e_{\omega}\right)
$$

where $\lambda_{\min }(\cdot)$ denotes the minimum eigenvalue and matrix $Q$ is given as:

$$
Q \triangleq\left[\begin{array}{cc}
\frac{1-2 k_{\alpha}-k_{\theta}}{2 k_{\theta}} & \sqrt{2}(1+L) \max \left\{1, k_{1}\right\}(1+c) \\
\sqrt{2}(1+L) \max \left\{1, k_{1}\right\} & 2 \min \left\{k_{1}, k_{2}\right\} \cos \left(e_{\theta}(0)\right)
\end{array}\right] .
$$

It remains to show that $Q$ is positive definite. It is reasonable to assume $k_{2}>k_{1}$, since $k_{2}$ has to be selected sufficiently large. A sufficient condition for $Q$ to have positive eigenvalues is

$$
k_{\alpha}>\frac{\max \left\{1, k_{1}\right\}^{2} \sec \left(e_{\theta}(0)\right) k_{\theta}(1+c)}{k_{1}}+\frac{1}{2}\left(k_{\theta}-1\right) .
$$

Then the smallest eigenvalue is:

$$
\begin{gathered}
\lambda_{\min }(Q)=-\frac{1}{4}+\frac{k_{\alpha}}{2 k_{\theta}}+(1+L) k_{1} \cos \left(e_{\theta}(0)\right)+\left[1 6 k _ { \theta } \left[2(1+c)^{2} \max \left\{1, k_{1}\right\}^{2} k_{\theta}\right.\right. \\
\left.\left.-(1+L) \cos \left(e_{\theta}(0)\right) k_{1}\left(1+2 k_{\alpha}-k_{\theta}\right)\right]+\left[1+2 k_{\alpha}+\left(4(1+L) k_{1} \cos \left(e_{\theta}(0)\right)-1\right) k_{\theta}\right]^{2}\right]^{1 / 2} .
\end{gathered}
$$

With respect to metric $d_{c}, V$ vanishes exponentially. Using the Comparison Lemma, we conclude for $\lambda \equiv \lambda_{\min }(Q)$ :

$$
\begin{aligned}
d_{c}\left(r(t), \varphi(t), e_{v}(t), e_{\theta}(t), e_{\omega}(t)\right) & \leq 2 k_{\theta} V\left(r(t), \varphi(t), e_{v}(t), e_{\theta}(t), e_{\omega}(t)\right) \\
\leq 2 k_{\theta} V(0) e^{-\lambda t} & \leq 2 k_{\theta} d_{c}\left(r(0), \varphi(0), e_{v}(0), e_{\theta}(0), e_{\omega}(0)\right) e^{-\lambda t}
\end{aligned}
$$

\section{Acceleration Perturbations}

This Section will establish the ultimate boundedness of the closed loop system (2)-(5)-(6) with respect to perturbations on the inputs $(a, \alpha)$, in the topology 
induced by (4). The bound on the state of the closed loop system is expressed in terms of a $\mathcal{K}$ class function of the magnitude of the disturbances. This implies that the system is (locally) input-to-state stable in the particular topology. Let the closed loop system (2)-(6) be perturbed by acceleration disturbances $\delta \triangleq\left(\delta_{a}, \delta_{\alpha}\right)^{T}$ :

$$
\begin{aligned}
\dot{r} & =\left(v+e_{v}\right) \cos \left(e_{\theta}+\varphi\right) \\
\dot{\varphi} & =\frac{v+e_{v}}{r} \sin \left(e_{\theta}+\varphi\right) \\
\dot{e}_{v} & =a+\delta_{a} \\
\dot{e}_{\theta} & =-k_{\omega} e_{\theta}+e_{\omega} \\
\dot{e}_{\omega} & =-e_{\theta}-k_{\alpha}+\delta_{\alpha}
\end{aligned}
$$

The time derivative of the Lyapunov function (12) will have two new terms:

$$
\begin{aligned}
\dot{V}=-\frac{k_{\theta}-1}{2 k_{\theta}}( & \left.e_{\theta}-e_{\omega}\right)^{2}-\frac{1+2 k_{\alpha}-k_{\theta}}{2 k_{\theta}}\left(e_{\theta}^{2}+e_{\omega}^{2}\right)+\frac{e_{v} \sin (2 \varphi)\left[\sin \left(\varphi+e_{\theta}\right)-\sin \varphi\right]}{r} \\
& +2 k_{1} \cos e_{\theta} \sin ^{2} \varphi-k_{1} \sin (2 \varphi) \sin e_{\theta}-2 k_{2} e_{v}^{2} \\
& -2 e_{v} r \sec ^{3} \varphi \sin ^{2}\left(\frac{e_{\theta}}{2}\right)-2 k_{1} r^{2} \cos e_{\theta} \sec ^{4} \varphi+2 e_{v} \delta_{a}+\frac{e_{\omega} \delta_{\alpha}}{k_{\theta}}
\end{aligned}
$$

and can be bounded from above as follows:

$$
\dot{V} \leq-\lambda d_{c}^{2}+2 e_{v} \delta_{a}+\frac{e_{\omega} \delta_{\alpha}}{k_{\theta}} \leq-\lambda d_{c}^{2}+\sqrt{2} \max \left\{2, \frac{1}{k_{\theta}}\right\} d_{c}\|\delta\|_{2} .
$$

For a constant parameter $\zeta \in(0,1)$, we can then have:

$$
\dot{V} \leq-\lambda(1-\zeta) d_{c}^{2}+\left(\sqrt{2} \max \left\{2, \frac{1}{k_{\theta}}\right\}\|\delta\|_{2}-\lambda \zeta d_{c}\right) d_{c}
$$

which is negative, provided that $d_{c} \geq \frac{\sqrt{2} \max \left\{2, \frac{1}{k_{\theta}}\right\}}{\lambda \zeta}\|\delta\|_{2}$. Treating (14) as a perturbed system, we have that:

$$
d_{c} \leq d_{c}(0) e^{-\lambda t}+\frac{\sqrt{2} \max \left\{2, k_{\theta}^{-1}\right\}}{\lambda \zeta}\|\delta\|_{2}
$$

implying that (14) is ISS with respect to the input $\delta$ and the norm induced by the metric $d_{c}$.

\section{Numerical Validation}

In this section we verify the ISS properties of the dynamic model (2) with respect to the metric chosen. In the simulation scenario, the initial conditions for the system are set to $(x, y, \theta, v, \omega)=\left(0.1,0.1,-\frac{\pi}{2}, 0,0\right)$ (Figure 3). 
The controller gains were set to $k_{1}=1, k_{\omega}=5, k_{2}=100 k_{1}$, and $k_{\theta}-2$. The system is perturbed by sinusoidal acceleration disturbances of the form $\delta_{a}=50 \sin (100 t)$ and $\delta_{\alpha}=50 \cos (100 t)$. Under these initial conditions and disturbances, the control scheme proves to be robust, ensuring convergence of the system state to the origin. The acceleration disturbances force the vehicle to chatter along its path however stability is maintained.

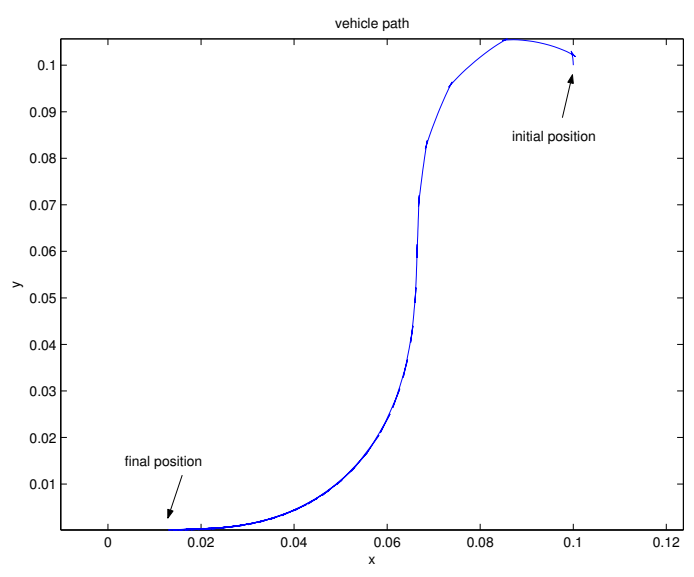

Fig. 3. Vehicle path.

Figures 4 and 5 show explicitly the effect of these disturbances on the position and velocity, errors of the vehicle respectively. Figure 6 shows the evolution of the metric $d_{c}$ along the trajectory of the vehicle, indicating clearly the initial transient phase where it the metric is decreasing and then the steady state, where it is ultimately bounded.

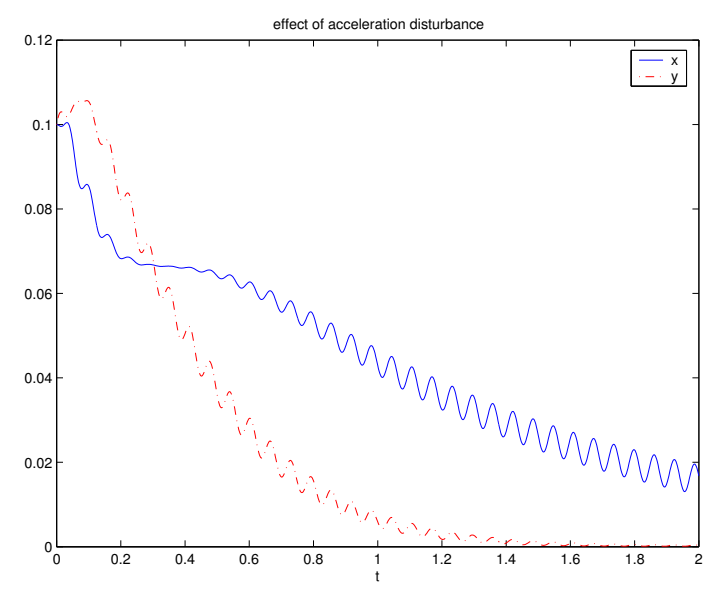

Fig. 4. The effect of acceleration disturbances on position.

\section{Conclusion}

In this paper we establish a local ISS property with respect to acceleration disturbances, for unicycle dynamics typically describing nonholonomic vehi- 


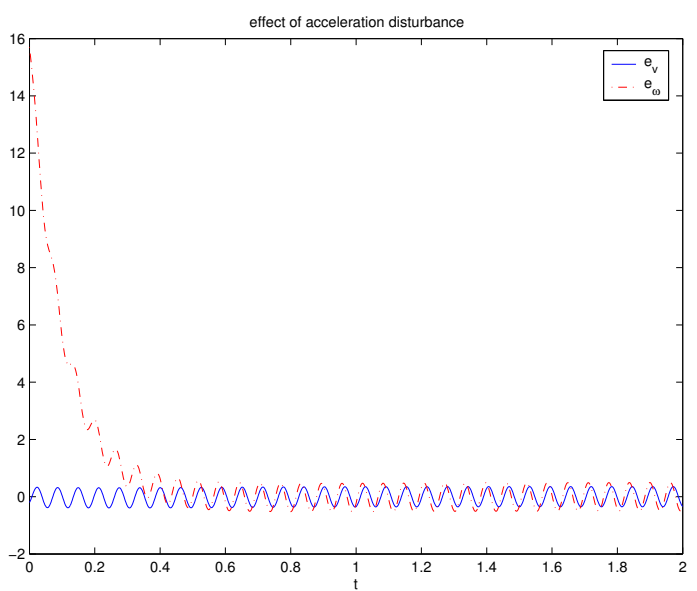

Fig. 5. The effect of acceleration disturbances on velocity errors.

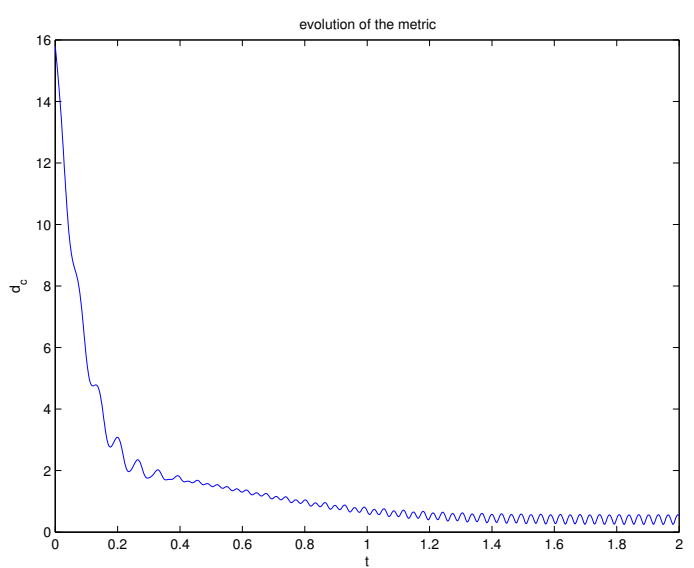

Fig. 6. The evolution of the metric along the trajectory.

cles. This is made possible in a topology induced by a particular nonholonomic metric, after the application of a (discontinuous) feedback input transformation. Beyond possible applications of the ISS property to the stability and robustness analysis of vehicle formations, this result shows that in a particular topology and with an appropriate choice of input transformation, systems that are not feedback linearizable or smoothly stabilizable may still enjoy ISS properties. To this point, it remains unclear whether the kinematic subsystem can also be rendered ISS. It seems though, that it is not the nonholonomic nature of the system, but rather the way in which inputs are introduced into the model equations that is more critical to establishing ISS properties with respect to these signals. 


\section{References}

[1] E. Sontag, On the input-to-state stability property, European Journal of Control 1 (1995) 24-36.

[2] A. Teel, L. Moreau, D. Nešić, Input-to-state set stability of pulse width modulated systems with disturbances, Systems \& Control Letters 51 (1) (2004) $23-32$.

[3] H. G. Tanner, G. J. Pappas, V. Kumar, Input-to-state stability on formation graphs, in: Proceedings of the IEEE International Conference on Decision and Control, Las Vegas, NV, 2002, pp. 2439 -2444.

[4] H. G. Tanner, G. J. Pappas, V. Kumar, Leader-to-formation stability, IEEE Transactions on Robotics and Automation(to appear).

[5] R. Brockett, Hybrid models for motion control systems, in: H. Trentelman, J. Willems (Eds.), Perspectives in Control, Birkhauser, Boston, 1993, pp. 2954 .

[6] I. Kolmanovsky, N. McClamroch, Developments in nonholonomic control problems, IEEE Control Systems (1995) 20-36.

[7] A. Bloch, Nonholonomic Mechanics and Control, Vol. 24 of Interdisciplinary Applied Mathematics, Springer, New York, 2003.

[8] E. Sontag, Smooth stabilization implies coprime factorization, IEEE Transactions on Automatic Control 34 (1989) 435-443.

[9] J.-P. Laumond, P. Souéres, Metric induced by the shortest paths for a car-like mobile robot, in: IEEE/RSJ International Conference on Intelligent Robots and Systems, 1993, pp. 1299-1304.

[10] P. Souéres, J.-P. Laumond, Shortest paths synthesis for a car-like robot, IEEE Transactions on Automatic Control 41 (5) (1996) 672-688.

[11] A. K. Das, R. Fierro, V. Kumar, J. P. Ostrowski, J. Spletzer, C. J. Taylor, A vision-based formation control framework, IEEE Transactions on Robotics and Automation 18 (5) (2002) 813-825.

[12] G. J. Pappas, K. J. Kyriakopoulos, Modeling and feedback control of nonholonomic mobile vehicles, in: Proceedings of the 1992 IEEE Conference in Decision and Control, 1992, pp. 241-244.

[13] E. D. Sontag, The ISS philosophy as a unifying framework for stability-like behavior, in: F. L.-L. A. Isidori, W. Respondek (Eds.), Nonlinear Control in the Year 2000, Volume 2, Lecture Notes in Control and Information Sciences, Springer-Verlag, Berlin, 2000, pp. 443-468. 